\title{
Molecular cloning of a phosphoenolpyruvate carboxylase cDNA from tropical epiphytic CAM orchid
}

\author{
C.R. LI, X.B. ZHANG* and C.S. HEW \\ Department of Biological Sciences, National University of Singapore, \\ 10 Kent Ridge Crescent, Singapore 119260
}

\begin{abstract}
A full-length cDNA encoding phosphoenolpyruvate carboxylase (PEPC) was isolated from tropical epiphytic CAM orchid Mokara Yellow. The cDNA designated as Mpepcl is $3450 \mathrm{bp}$ in length with an open reading frame of $2862 \mathrm{bp}$ encoding 954 amino acids. The deduced amino acid sequence of Mpepcl shows $83 \%$ identity with pepc2 of sorghum, $82 \%$ with pepcl and pepc2 of maize and $81 \%$ with pepc of Arabidopsis thaliana. RT-PCR analysis showed that Mpepcl was expressed in mature leaves, immature leaves, and aerial roots of $M$. Yellow. No expression was detected in the flower.
\end{abstract}

Additional key words: amino acid sequence, Mokara Yellow, RACE.

Phosphoenolpyruvate carboxylase (PEPC) catalyses the carboxlylation of phosphoenolpyruvate to oxaloacetate and $\mathrm{Pi}$, the first step in the photosynthetic assimilation of $\mathrm{CO}_{2}$ in CAM and $\mathrm{C}_{4}$ plants. It has been proposed that plant PEPCs are encoded by a small multigene family and each member within a gene family encodes a distinct isoform which is associated with specific physiological functions (Lepiniec et al. 1994). In maize and sorghum, three PEPC isoforms were identified (Yanagisawa et al. 1988, Cretin et al. 1991, Kawamura et al. 1992, Lepiniec et al. 1993). So far most of the molecular studies on PEPC had been carried out on $\mathrm{C}_{3}$ or $\mathrm{C}_{4}$ plants. Here we report the cloning of a full-length cDNA encoding PEPC from a tropical epiphytic CAM orchid hybrid Mokara Yellow (Arachnis hookeriana $\times$ Ascocenda Madame Kenny).

Plants of $M$. Yellow were obtained from a local nursery. They were planted in pots of sand in growth chambers with $150-200 \mu \mathrm{mol} \mathrm{m} \mathrm{m}^{-2} \mathrm{~s}^{-1}$ of photosynthetically active radiation at leaf height (Thorlux lamp, $400 \mathrm{~W}, 240 \mathrm{~V}, 50 \mathrm{~Hz}$; Redditch, UK), 70 - $80 \%$ relative humidity, $30 / 25^{\circ} \mathrm{C}$ day/night temperature and $12-\mathrm{h}$ photoperiod. Plants were watered daily with half strength Hoagland's solution B.
Total RNA was isolated from different tissues at different developmental stages using NucleoSpin RNA plant kit (Macherey-Nagel, Düren, Germany). Based on the available published sequences, the following primers were synthesized: sense primer P1: 5'-TCT TGG ATG GGT GGT GAT CGT GAT GG-3' and antisense primer P2: 5'-AAA GAT CCA AGG GAT TGC ACG GAG TGA-3'. RT-PCR was performed with one-step RT-PCR kit (BD Biosciences, Palo Alto, CA, USA) according to the manufacturer's instructions. The amplified $1475 \mathrm{bp}$ fragment was cloned into the PTZ57R vector with InsT/Aclone PCR Cloning Kit (Fermentas, Vilnius, Lithuania). The identity of the amplified product was confirmed by sequencing.

The following gene specific primers were designed from the sequence of the $1475 \mathrm{bp}$ fragment to perform 5' and 3' RACE using the SMART RACE cDNA kit (BD Biosciences). GSP1: 5'-GGT GGA ACC TGT TGG AAG AGG AGG CGG TCC-3' and GSP2: 5'-GCA CGC CAT TCT GGC TTA GGA GAG ATT GGG-3'. The 5' RACE and 3' RACE products were cloned into the pTZ57R vector and sequenced. To generate full-length CDNA, primers were designed from the $5^{\prime}$ end of the 5' RACE product sequence and the 3' end of the

Received 14 February 2003, accepted 15 September 2003.

Abbreviations: PEPC - phosphoenolpyruvate carboxylase; RACE - rapid amplification of cDNA ends; RT-PCR - reverse transcriptase polymerase chain reaction.

* Author for correspondence; fax: $(+65)$ 7792486, e-mail: zhang xiaobs $(a)$ hotmail.com 
3' RACE product sequence. The template for this final PCR reaction was from the original first-strand RT-PCR step.

Results showed that the full-length cDNA encoding PEPC from $M$. Yellow is 3450 bp in length with an open reading frame of 2862 bp encoding 954 amino acids. The deduced amino acid sequence of Mpepcl shared $83 \%$ identity with that of pepc2 from sorghum, $82 \%$ identity with those of pepcl and pepc2 from maize, pepcl from sorghum, pepc from rice and sugarcane, $81 \%$ with those of pepc from Arabidopsis thaliana and wheat (Table 1).

Table 1. Amino acid sequence identity of Mpepcl (accession number AF530570) with PEPC from other plant species calculated by Align $X$ program of sequence analysis software Vector NTI (InforMax, Bethesda, Maryland, USA).

\begin{tabular}{lll}
\hline Gene name & Accession number & Identity [\%] \\
\hline Sorghum-pepc2 & X59925 & 83 \\
Sorghum-pepcl & X55664 & 82 \\
Maize-pepc1 & AB012228 & 82 \\
Maize-pepc2 & X61489 & 82 \\
Rice-pepc & AF271995 & 82 \\
Sugarcane-pepc & M86661 & 82 \\
Vanilla-pepc & X87148 & 82 \\
Arabidopsis thaliana-pepc & AY074346 & 81 \\
Wheat-pepc & AJ007705 & 81 \\
\hline
\end{tabular}

RT-PCR cannot be used to quantify the level of gene expression. However, in this study, equal amounts of RNA were used for each RT-PCR reaction using the same RT-PCR kit and equal amounts of RT-PCR product

\section{References}

Cretin, C., Santi, S., Keryer, E., Lepiniec, L.., Tagu, D., Vidal, J., Gadal. P.: The phosphoenolpyruvate carboxylase gene family in Sorghum: promoter structure, amino acid sequences and expression of genes. - Gene 99: 87-94, 1991.

Cushman, J.C., Meyer, G., Michalowski, C.B., Schmitt, J.M., Bohnert, H.J.: Salt stress leads to differential expression of two isogenes of phosphoenolpyruvate carboxylase during Crassulacean acid metabolism induction in the common ice plant. - Plant Cell 1: 715-725, 1989.

Kawamura, T., Shigesada, K., Toh, H., Okumura, S., Yanagisawa, S., Izui, K.: Molecular evolution of phosphoenolpyruvate carboxylase for $\mathrm{C}_{4}$ photosynthesis in maize: comparison of its cDNA sequence with a newly isolated cDNA encoding an isozyme involved in the anaplerotic function. - J. Biochem. 112: 147-154, 1992. were loaded on agarose gels. The relative amounts of RT-PCR product (as shown by the intensity of signal on agarose gel) might be a good indicator for the relative levels of mRNA expression in different tissues of plants. The highest level of Mpepcl expression was found in mature leaves, although it was detectable in immature leaves and aerial roots of $M$. Yellow (Fig. 1). No expression was detected in the flowers. There is evidence that in all plants a constitutively expressed PEPC isoform $\left(\mathrm{C}_{3}\right.$ isoform, which catalyses mainly anaplerotic reactions) exists (Lepiniec et al. 1991) and in CAM plants a CAM-specific PEPC isoform is expressed which is responsible for primary $\mathrm{CO}_{2}$ fixation of this photosynthetic pathway (Cushman et al. 1989). The strong expression of Mpepcl in the leaves (CAMperforming organ) suggests that $M p e p c l$ might be the CAM-specific PEPC isoform in the constitutive CAM plant $M$. Yellow. Further study is needed on other PEPC isoforms in this plant.

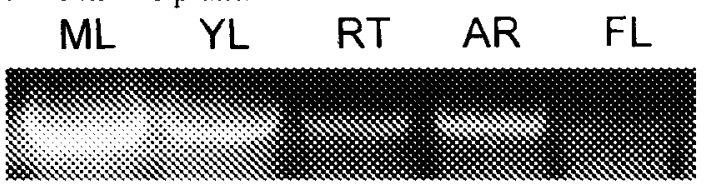

Fig. 1. RT-PCR analysis of Mpepcl expression in different tissues of $M$. Yellow. ML - mature leaves, YL - young leaves, RT - aerial root tips, AR - aerial roots without root tips, FL - flower. About $0.2 \mu \mathrm{g}$ of total RNA was used for each reaction. $0.01 \mathrm{~cm}^{3}$ RT-PCR product was loaded on each lane of $1 \%$ agarose gel. The following primers were used for the RT-PCR reaction: RT1: 5'-GAG GCA GCC CCT GCA GCT GTG G-3' and RT2: 5'-TCA TAC AGA GCA GCG ATT CCT GAG TTG CCT -3 '.

L.epiniec, L., Keryer, E., Philippe, H., Gadal, P., Cretin, C.: Sorghum phosphoenolpyruvate carboxylase gene family: structure, function and molecular evolution. - Plant mol. Biol. 21: 487-502, 1993

Lepiniec, L., Santi, S., Keryer, E., Amiet, V., Vidal, J., Gadal, P., Cretin, C.: Complete nucleotide sequence of one member of the sorghum phosphoenolpyruvate carboxylase gene family. - Plant mol. Biol. 17: 1077-1079, 1991.

Lepiniec, L., Vidal, J., Chollet, R., Gadal, P., Cretin, C.: Phosphoenolpyruvate carboxylase: structure, regulation and evolution. - Plant Sci. 99: 111-124, 1994.

Yanagisawa, S., Izui, K., Yamaguchi, Y., Shigesada, K., Katsuki, H.: Further analysis of cDNA clones for maize phosphoenolpyruvate carboxylase involved in $\mathrm{C}_{4}$ photosynthesis. - FEBS Lett. 229: 107-110, 1988. 